Mediterránea Ser. Biol. (1988), n. ${ }^{\circ}$ 10. Pág. 45-61

\title{
UNA FUNCIÓN DE CRECIMIENTO FLEXIBLE PARA DESCRIBIR DIFERENTES TIPOS DE SERIES DENDROCRONOLÓGICAS: GENERALIZACIÓN DE LA FUNCIÓN DE RICHARD
}

\author{
por \\ C. A. GRACIA ${ }^{1}$ Y J. J. IBÁÑEZ 1
}

\section{RESUMEN}

Se proponen modificaciones a la función de Richard (1959), de manera que resulta posible describir el crecimiento indeterminado de los árboles. En el trabajo se discute el modelo biológico que constituye la base teórica de la función propuesta. Se presentan numerosos ejemplos de aplicación que sirven, al mismo tiempo, para discutir la relación existente entre los parámetros de la función así como la estabilidad de las estimaciones que se consiguen en el algoritmo que utiliza el programa GROWTH desarrollado por uno de los autores.

Palabras clave: crecimiento, función de Richard, dendrocronología.

\section{SUMMARY}

A flexible growth function to describe dendrochronological series: a generalization of the $\mathrm{Ri}$ chard's function.

Some modifications to the Richards function are proposed in order to make it suitable for the description of undeterminate growth. The biological model wich remains in the basis of the proposed function is discussed. Some practical cases allows us to present the relationships among the parameters of the function as well as the stability of the estimations obtained with GROWTH, a computer program implemented by one of the authors.

Key words: growth, Richard's function, dendrochronology.

\section{INTRODUCCIÓN}

El espesor de los anillos de crecimiento de los árboles se puede considerar como el resultado de la interacción de dos tipos de componentes sobre la plan-

(1) Departamento de Ecología, Facultad de Biología. Universidad de Barcelona. 
ta. Por un lado las componentes endógenas, controladas por la propia planta, que responden a patrones genéticos y por otra parte las componentes exógenas principal, pero no exclusivamente, de tipo climático que fluctúan irregularmente y a las que cada planta responde con más o menos inercia.

El grosor de un anillo de crecimiento formado en un año particular $t$, dependerá de los factores externos a los que el árbol se ha visto sometido durante el año anterior (y eventualmente los años anteriores) de modo que condiciones favorables se traducen en una mayor acumulación de productos resultantes de la fotosíntesis $\mathrm{y}$, por lo tanto, en un mayor espesor del anillo. Pero además de estos factores externos juegan un papel importante los factores que hemos llamado endógenos. En efecto, en el año particular t, los árboles más jóvenes que se encuentran en su fase juvenil, presentan una tendencia mayor al crecimiento de la que presentan árboles más maduros de la misma población, exactamente igual que ocurre con otros muchos organismos, animales o plantas.

La varianza de los espesores de los anillos no es homogénea en el tiempo ya que, en ausencia de perturbaciones externas, tiende a decrecer al incrementarse la edad del árbol. El espesor medio de los anillos, la desviación respecto de la media y la estructura de autocorrelación varían marcadamente dependiendo de las condiciones de su lugar de implantación, así como de la edad del árbol. (FRITTS, 1976).

En consecuencia, la correcta interpretación de una serie dendrocronológica requiere diferenciar los efectos debidos a estos dos grupos de componentes. El procedimiento habitual consiste en ajustar la curva de crecimiento del árbol a alguna función matemática conocida para, más tarde, calcular los índices de crecimiento como cociente entre los valores reales (medida del espesor del anillo) y los estimados (valor en la función de ajuste elegida).

Este procedimiento, conocido como estandarización, permite la comparación de las distintas series cuyos valores, transformados en índices, presentan una varianza más homogénea en el tiempo, un valor análogo para todos los índices medios, la mínima desviación de los índices respecto del valor medio, además de haber eliminado la parte de autocorrelación debida a la tendencia impuesta por el crecimiento.

Una gran variedad de funciones tales como exponenciales negativas (FRITTS 1976, BERGER ET AL 1979, GRAYBILL 1981, GÉNOVA 1987), logarítmicas (ANIOL 1983, ZUCHINNI y HIEMSTRA 1983), de medias móviles (STOKES y SMILEY 1968, BAILLIE 1982), polinomiales (FRITTS 1976, BAILLIE 1982) y multitud de otras transformaciones (GUTIÉRREZ, 1987) se han utilizado con el fin de recoger la función de crecimiento de las distintas poblaciones de diferentes especies.

Las funciones matemáticas utilizadas son, con frecuencia, funciones empíricas que se ajustan a los datos pero, cuyos parámetros, carecen de sentido biológico. Un problema accesorio es que, con frecuencia, se han de utilizar distintas funciones para ajustar árboles de una misma especie e incluso de una misma población. Por otra parte, la correcta aplicación de algunas de estas funciones obliga a desechar en numerosos casos, y siempre de forma subjetiva, una parte de los valores que no son aptos para el ajuste seleccionado. Un buen ejemplo lo constituye el caso de los valores - años-iniciales en los ajustes a funciones exponenciales negativas (GÉNOVA, 1987). 
Una consecuencia negativa que se deriva de tal proceder es la pérdida de la información contenida en las curvas de crecimiento de los diferentes árboles. El estudio comparativo de curvas de individuos que viven en una misma masa forestal, nos aporta información sobre los efectos de la competencia intraespecífica (GRACIA E IBÁÑNEZ, 1985) a condición de que la función utilizada para el ajuste sea la misma en todos ellos, a fin de hacer posible la comparación entre los parámetros.

RICHARD (1959) propuso una función de tipo muy general, capaz de describir el crecimiento de organismos muy diferentes. Esta función que engloba a otras tan conocidas como la de Gompertz, la logística o la de Von Bertalanffy como casos particulares tiene, sin embargo, el inconveniente de que está acotada por una asíntota superior horizontal (crecimiento determinado) que la hace poco adecuada para describir el crecimiento de tipo indeterminado que presentan los árboles al añadir nuevos anillos por la parte externa del xilema. Desde el punto de vista matemático esta función ha sido estudiada exhaustivamente por CAUSTON ELIAS y HADLEY (1978) y por CAUSTON y VENUS (1981).

En el presente trabajo presentamos una generalización de la función de Richard, de tal modo que se suprime la limitación de la asíntota horizontal y la hace especialmente adecuada para describir el crecimient de los árboles.

\section{LA FUNCIÓN DEL CRECIMIENTO}

RICHARD (1959) propone definir el crecimiento de un organismo mediante una función del tipo:

$$
R_{t}=\alpha \cdot\left[1 \pm e^{(\beta-k t)}\right]^{-1 / v}
$$

donde $\mathbf{R}_{t}$ es el tamaño del individuo en el instante $t$. En el caso particular del crecimiento de los árboles, y por comodidad en la exposición posterior, consideraremos como tamaño el radio del tronco, medido a la altura estándar de 1.30 metros. $\alpha, \beta, \mathrm{k}$ y v son los cuatro parámetros de ajuste de la función tales que:

$$
\begin{array}{lllll}
\alpha & \mathrm{y} & \mathrm{k}>0 & \mathrm{y} & -1 \leq \mathrm{v}<\infty \quad \mathrm{v} \neq 0
\end{array}
$$

El parámetro $\alpha$ representa el valor de la ordenada en el origen de la asíntota superior que señala el límite del crecimiento (figura 1). El valor del parámetro $\beta$ se relaciona estrechamente con la localización de la curva en el eje del tiempo y resulta, en consecuencia altamente informativo a pesar de la opinión contraria de CAUSTON y VENUS (op. cit. pág. 145). En particular, podemos afirmar que, a medida que la curva se desplaza en dicho eje y, por ejemplo, el punto de inflexión se localiza más tarde, el valor de $\beta$ aumenta como queda de manifiesto comparando las funciones de la figura 2 cuyos parámetros estimados se resumen en la tabla 1 .

Cuando $\mathrm{v}=-1$ la función representa el caso particular de la función de Gompertz, en tanto que el valor de $\mathrm{v}=1$ representa el caso particular de la función logística. Así mismo la función de von Bertalanffy puede considerarse 


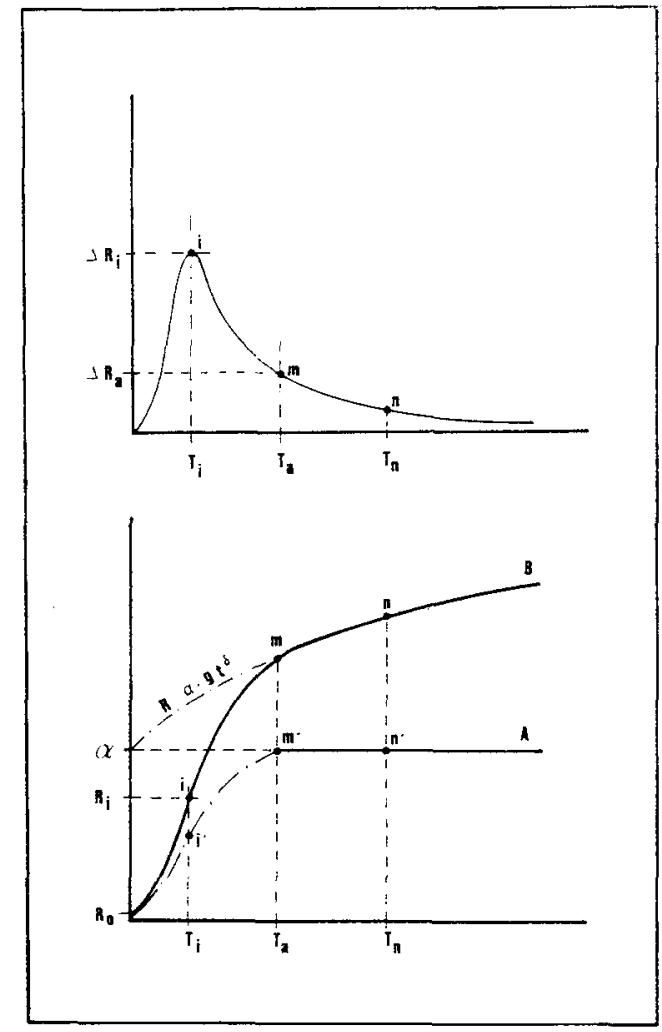

Figura 1.-El crecimiento de un árbol puede representarse como una serie de incrementos anuales (parte superior) o como una serie de crecimientos acumulados (parte inferior) que es la forma utilizada por la función de Richard (función A, parte inferior). En este caso el máximo incremento anual (i) se transforma en un punto de inflexión. La función (B), que corresponde a un crecimiento indeterminado, exige la introducción de los parámetros g y $\delta$ para describir la función alométrica que limita el crecimiento.

\begin{tabular}{lrrrrrrr} 
CORE & \multicolumn{1}{c}{$\alpha$} & \multicolumn{1}{c}{$\beta$} & $\gamma$ & $\delta$ & $\kappa$ & \multicolumn{1}{c}{$v$} & \multicolumn{1}{c}{$\mathrm{T} i$} \\
\hline A: PS-11-PB2 & 22.93 & -0.29 & 3.77 & 0.85 & 0.48 & 0.46 & 1.01 \\
B: PU-24-M & 23.50 & -0.09 & 1.65 & 0.91 & 0.11 & -0.67 & 2.82 \\
C: PS-13-PB2 & 22.38 & 0.04 & 1.12 & 0.78 & 0.21 & -0.56 & 2.95 \\
D: PS-3-PB2 & 26.56 & 1.45 & 0.80 & 0.76 & 0.27 & 0.66 & 6.91 \\
E: PP-17-GV & 18.89 & 10.59 & 2.50 & 0.92 & 0.63 & 3.08 & 15.02 \\
F: PU-9-M & 7.51 & 20.93 & 0.99 & 0.97 & 1.02 & 4.33 & 19.08
\end{tabular}

Tabla 1.-Valores estimados de los parámetros de la función de crecimiento de los árboles representados en la figura 2. Compárese en particular el momento en el que se produce la inflexión en el crecimiento $T_{\mathrm{i}}$ con el valor del parámetro $\beta$. A, C y D: Pinus sylvestris, Puertos de Beceite, Tarragona. B. y F: Pinus uncinata; Masella, Gerona. E: Pinus pinea, Gavarres, Gerona. 
TABLA 2

g

$\delta$

Pinus sylvestris

Beceite
Parcela 1

23

0.71

0.859

$\begin{array}{lll}25 & 1.281 & 0.945\end{array}$

$\begin{array}{lll}26 & 1.250 & 0.950\end{array}$

$\begin{array}{lll}27 & 1.207 & 0.962\end{array}$

$\begin{array}{lll}27 & 1.232 & 0.976\end{array}$

$\begin{array}{lll}27 & 1.599 & 0.992\end{array}$

$\begin{array}{lll}27 & 2.198 & 0.920\end{array}$

Beceite

41

0.086

0.961

Parcela 2

45

0.184

0.976

46

0.097

0.952

47

1.651

0.521

48

0.121

0.949

51

2.008

0.920

5

0.603

0.994

52

0.913

0.988

52

2.664

0.577

53

0.359

0.996

53

0.801

0.934

53

0.912

0.989

53

$\begin{array}{ll}1.221 & 0.998\end{array}$

$\begin{array}{lll}53 & 2.547 & 0.815\end{array}$

$\begin{array}{llll}54 & 0.421 & 0.981\end{array}$

$\begin{array}{lll}54 & 0.478 & 0.963\end{array}$

$\begin{array}{lll}54 & 0.735 & 0.973\end{array}$

$\begin{array}{lll}54 & 0.955 & 0.995\end{array}$

$\begin{array}{lll}54 & 1.007 & 0.987\end{array}$

$\begin{array}{lll}54 & 1.214 & 0.994\end{array}$

$\begin{array}{lll}54 & 1.738 & 0.679\end{array}$

$\begin{array}{lll}55 & 0.331 & 0.947\end{array}$

$\begin{array}{lll}55 & 0.827 & 0.901\end{array}$

$\begin{array}{lll}55 & 1.283 & 0.823\end{array}$

$\begin{array}{lll}55 & 0.331 & 0.947\end{array}$

$\begin{array}{llll}56 & 0.460 & 0.969\end{array}$

$\begin{array}{lll}56 & 0.821 & 0.991\end{array}$

$\begin{array}{lrrr}\text { Cebollera } & 257 & 0.848 & 0.910\end{array}$

$\begin{array}{lll}258 & 0.886 & 0.973\end{array}$

Pinus uncinata

$\begin{array}{lll}265 & 1.206 & 0.920\end{array}$

Masella

$\begin{array}{lll}51 & 3.397 & 0.690\end{array}$

$\begin{array}{lll}52 & 3.445 & 0.938\end{array}$

$\begin{array}{lll}61 & 1.355 & 0.931\end{array}$

$\begin{array}{lll}62 & 1.042 & 0.979\end{array}$

$\begin{array}{lll}78 & 0.828 & 0.997\end{array}$

$\begin{array}{lll}78 & 2.063 & 0.857\end{array}$

$\begin{array}{lll}79 & 1.189 & 0.954\end{array}$ 


\begin{tabular}{|c|c|c|c|c|}
\hline ESPECIE & LOCALIDAD & EDAD & g & $\delta$ \\
\hline & & 79 & 1.994 & 0.988 \\
\hline & & 86 & 0.599 & 0.936 \\
\hline & & 87 & 0.557 & 0.977 \\
\hline & & 87 & 0.987 & 0.971 \\
\hline & & 88 & 1.013 & 0.977 \\
\hline & & 89 & 1.296 & 0.997 \\
\hline & & 93 & 0.518 & 0.992 \\
\hline & & 95 & 1.305 & 0.844 \\
\hline & & 97 & 0.459 & 0.977 \\
\hline & & 100 & 1.570 & 0.914 \\
\hline & & 100 & 1.073 & 0.957 \\
\hline & & 107 & 1.127 & 0.979 \\
\hline & & 108 & 0.691 & 0.991 \\
\hline & & 108 & 1.261 & 0.951 \\
\hline & & 110 & 0.595 & 0.985 \\
\hline & & 111 & 0.691 & 0.991 \\
\hline & Molina & 44 & 2.589 & 0.969 \\
\hline & & 49 & 1.306 & 0.935 \\
\hline & & 51 & 0.300 & 0.966 \\
\hline & Vallibierna & 50 & 2.599 & 0.972 \\
\hline & & 51 & 1.309 & 0.998 \\
\hline & Gossol & 91 & 0.448 & 0.786 \\
\hline \multirow[t]{2}{*}{ Pinus halepensis } & Salou & 23 & 0.223 & 0.959 \\
\hline & & 23 & 0.390 & 1.000 \\
\hline Pinus pinea & Gavarres & 62 & 2.500 & 0.922 \\
\hline Pseudotsuga menz. & Montseny & 15 & 5.214 & 0.826 \\
\hline \multirow[t]{2}{*}{ Quercus pubescens } & Tavertet & 58 & 1.741 & 1.001 \\
\hline & & 60 & 2.735 & 0.919 \\
\hline Quercus ilex & Cáceres & 91 & 1.651 & 0.933 \\
\hline Fagus sylvática & Grevulosa & 107 & 2.540 & 0.988 \\
\hline
\end{tabular}

Tabla 2.- Valores de los parámetros g y $\delta$, que describen el crecimiento asintónico, en árboles de diferentes especies y edades. Nótese que prácticamente todos los valores de $\delta$ quedan comprendidos entre 0.5 y 1 , como corresponde al modelo propuesto.

como un caso particular de esta función como han demostrado CAUSTON y VENUS (1981).

Los parámetros $\beta, \mathrm{k}$ y v presentan un grado notable de correlación entre ellos, como veremos más adelante, pero permiten una flexibilidad notable y, en consecuencia, la función resulta muy sensible cuando se ajustan series de crecimiento reales. Sin embargo, a pesar de las particulares propiedades de la función de Richard, resulta difícil describir el crecimento indeterminado que presentan los árboles, ya que al añadir cada año los anillos de crecimiento por la parte exterior del xilema se produce un permanente crecimiento del radio. En realidad este incremento de anillos funcionales por la parte externa del xilema viene compensado por la paralización de la actividad conductora de los 
anillos interiores, más viejos, que dejan de ser funcionales, dando origen al duramen. No obstante, puesto que el diámetro del tronco constituye un parámetro fundamental en el estudio de las relaciones alométricas del árbol, una correcta descripción de su incremento resulta fundamental a la hora de elaborar planes de ordenación y aprovechamiento forestal sin contar con el problema específico y bien definido de la estandarización en dendrocronología al que hemos hecho referencia en la introducción. Éstas son algunas de las razones que justifican el introducir modificaciones en la función de Richard de modo que permita describir patrones de crecimiento indeterminado.

Para entender el sentido de la función que proponemos consideraremos, de modo intuitivo, que el crecimiento de un árbol se puede explicar como el efecto aditivo de dos componentes. Por una parte el árbol se ve sometido a un patrón de crecimiento sigmoide que se manifiesta en función de la edad de los individuos y que viene impuesto por el desfase que se produce entre la formación del aparato radicular y la formación del aparato asimilador. Como consecuencia, el crecimiento potencial de los individuos aumenta progresivamente hasta alcanzar un máximo incremento radial. En realidad, puesto que el xilema está constituido en su mayor parte por células muertas, es fácil entender que, una vez que se alcanza un desarrollo de las copas - si se prefiere, un índice foliar - tal que la capacidad evaporativa de la planta se equilibra con su disponibilidad hídrica y/o la extinción de la luz ya no permite la acumulación de mayor superficie foliar, se alcanza un equilibrio evaporativo tal que la sección transportadora de agua a un nivel determinado del tronco, ha de mantenerse aproximadamente constante.

Puesto que los nuevos anillos se añaden por la parte exterior del tronco y los que dejan de ser funcionales son los que se localizan más hacia el centro, es fácil darse cuenta de que, puesto que un anillo externo representa más superficie que uno interno de igual espesor, la constancia de la sección transportadora de agua se consigue reduciendo el espesor de cada nuevo anillo. En realidad equivale a considerar que el árbol fabrica la misma sección de madera cada año - fluctuaciones impuestas por el clima y otros factores externos aparte- pero la dispone en anillos más estrechos y de mayor radio. Si aceptamos provisionalmente este modelo de crecimiento, el espesor de los sucesivos anillos $t+1, t+2, \ldots, t+n$ a partir del año $t$ en el que el bosque alcanza su equilibrio evaporativo, se ajusta a una función del tipo:

$$
\mathrm{R}_{\mathrm{t}}=\mathrm{K}+\mathrm{gt}^{0.5}
$$

donde la constante $\mathrm{K}$ representa el crecimiento del árbol hasta el momento en el que éste alcanza la asíntota y que no es otra cosa que la función de Richard.

En realidad, puesto que asociado al aumento del diámetro se produce un incremento de la altura del árbol, existe una dificultad suplementaria para transportar el agua a una mayor altura, que se compensa aumentado proporcionalmente el área de la sección transportadora. Esto equivale a decir que el espesor del anillo tiende a ser tal que el área incrementada cada año es ligeramente superior a la del año anterior. 

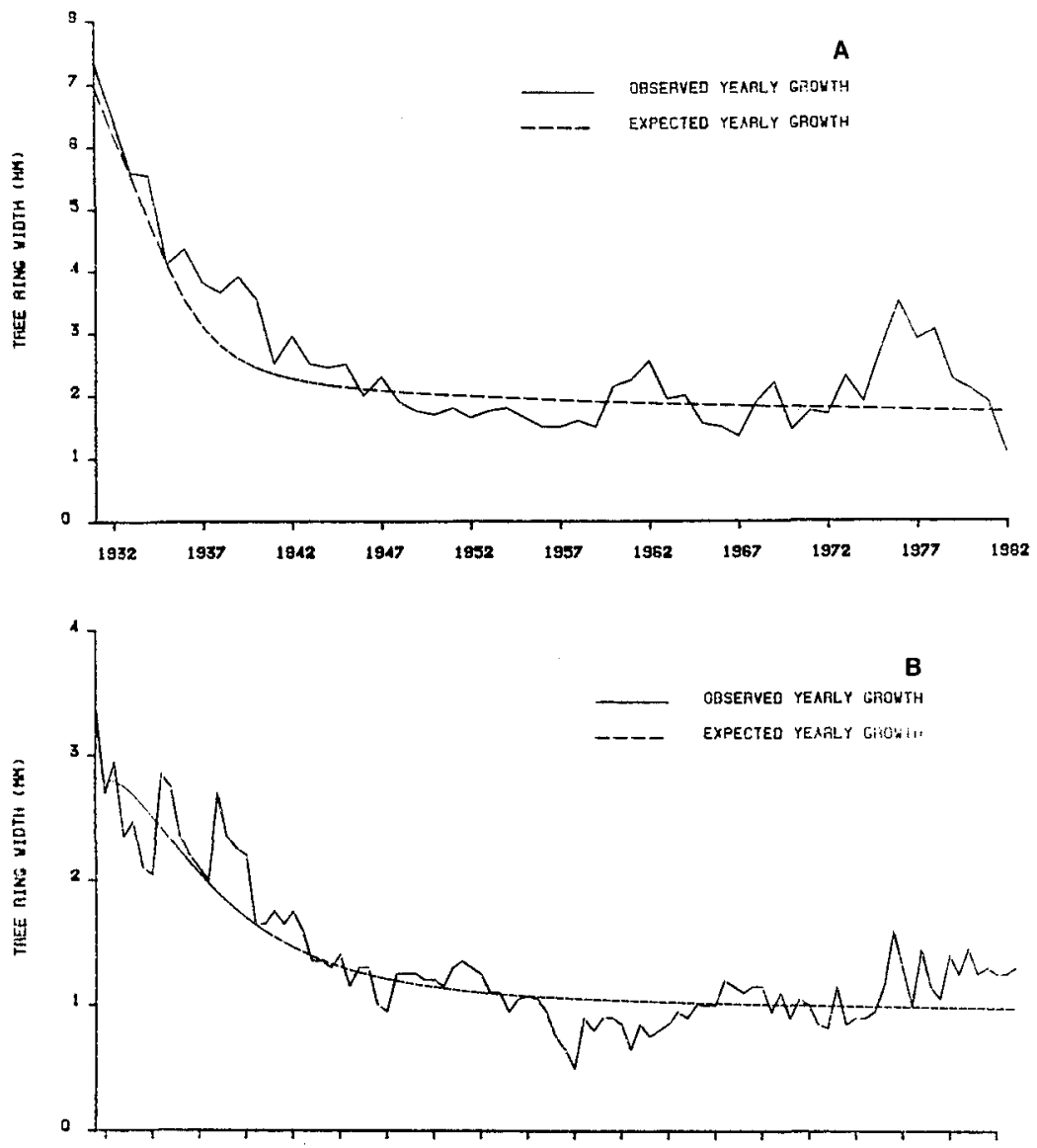

18 as t890 169519001965191019151920192519301935194019451950193519601565197019751560

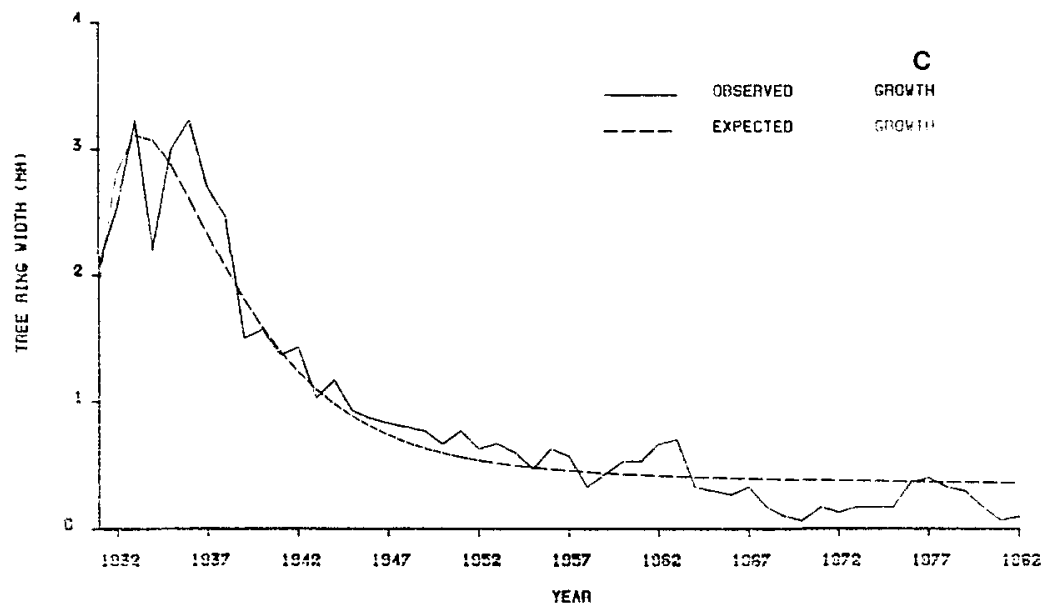



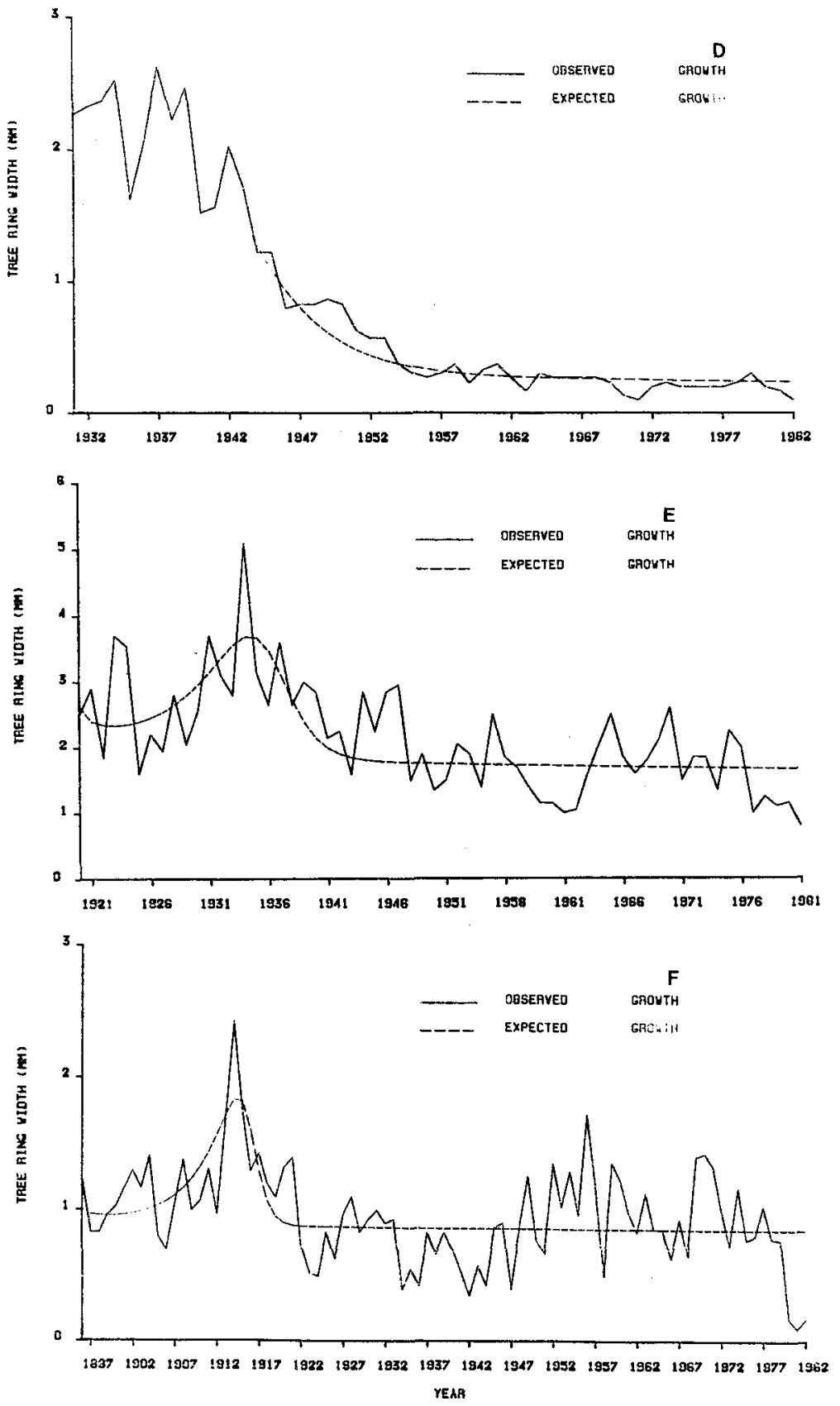

Figura 2.-Curvas de crecimiento anual de seis árboles del NE de España. Las diferencias en la «forma» de las diferentes curvas queda bien recogida en los parámetros de la función ajustada. (Ver tabla 1). A, C y D: Pinus sylvestris, Puertos de Beceite, Tarragona. B y F: Pinus uncinata, Masella, Gerona. E: Pinus pinea, Gavarres, Gerona. 
$\alpha$ $\beta$ $\varphi$ $\delta$ $\kappa$ v SM VM IND.

\begin{tabular}{|c|c|c|c|c|c|c|c|c|c|}
\hline S-PB-A1 & 48.637 & -0.437 & 1.297 & 0.962 & 0.315 & -0.183 & 0.202 & 0.220 & 0.953 \\
\hline S-PB-A2 & 64.750 & -0.010 & 1.149 & 1.000 & 0.215 & -0.419 & 0.184 & 0.299 & 1.029 \\
\hline S-PB-A4 & 22.733 & 0.297 & 2.000 & 1.000 & 0.505 & -0.490 & 0.209 & 1.114 & 1.377 \\
\hline S-PB-A5 & 22.216 & 3.149 & 0.187 & 1.000 & 0.713 & 1.346 & 0.274 & 0.143 & 1.141 \\
\hline S-PB-A6 & 25.390 & 2.099 & 0.230 & 1.000 & 0.608 & 0.716 & 0.258 & 0.094 & 1.185 \\
\hline S-PB-A7 & 43.066 & 0.054 & 1.281 & 0.945 & 0.334 & 0.199 & 0.230 & 0.398 & 1.034 \\
\hline S-PB-A 8 & 43.100 & -0.283 & 1.650 & 1.000 & 0.276 & -0.250 & 0.199 & 0.344 & 1.035 \\
\hline S-PB-A46 & 52.280 & -0.992 & 0.230 & 1.000 & 0.304 & 0.081 & 0.242 & 0.154 & 1.07 \\
\hline S-PB-A47 & 68.217 & 0.033 & 1.517 & 1.000 & 0.169 & -0.445 & 0.170 & 0.206 & 1.008 \\
\hline S-PB-A48 & 40.327 & -1.919 & 1.233 & 0.976 & 0.336 & 0.027 & 0.203 & 0.996 & 1.027 \\
\hline S-PB-A49 & 38.840 & -0.114 & 0.520 & 1.000 & 0.322 & 0.179 & 0.221 & 0.253 & 1.140 \\
\hline S-PB-A50 & 31.083 & -0.468 & 0.267 & 1.000 & 0.352 & 0.153 & 0.259 & 0.224 & .017 \\
\hline S-PB-A51 & 32.216 & -0.299 & 0.062 & 1.281 & 0.367 & -0.247 & 0.275 & 0.075 & 1.133 \\
\hline S-PB-A80 & 41.350 & 0.146 & 1.450 & 1.000 & 0.175 & -0.684 & 0.165 & 0.159 & 1.040 \\
\hline S-PB-A82 & 27.701 & -1.184 & 1.257 & 1.003 & 0.412 & 0.059 & 0.214 & 0.185 & 0.964 \\
\hline S-PB-A83 & 26.031 & 2.856 & 1.599 & 0.992 & 0.574 & 0.986 & 0.164 & 0.198 & 1.016 \\
\hline S-PB-A 85 & 804 & 5.025 & 0.896 & 1.008 & 0.602 & 2.249 & 0.281 & 0.254 & 1.053 \\
\hline S-PB-A86 & 36.427 & -0.481 & 0.263 & 1.000 & 0.368 & 0.098 & 0.356 & 0.127 & 0.955 \\
\hline S-PB-A86 & 14.541 & 0.764 & 0.711 & 0.859 & 0.698 & 0.265 & 0.262 & 0.077 & 0.997 \\
\hline S-PB-A88 & .697 & 0.120 & 0.267 & 1.000 & 0.291 & -0.471 & 0.259 & 0.033 & 1.086 \\
\hline B-A 120 & 54.483 & 0.094 & 1.333 & 1.000 & 0.167 & -0.665 & 0.204 & 0.254 & 1.032 \\
\hline S-PB-A 121 & 44.607 & 0.110 & 2.198 & 0.920 & 0.183 & -0.645 & 0.163 & 0.276 & 1.076 \\
\hline S-PB-A3 & 33.358 & 1.217 & 0.199 & 1.003 & 0.220 & 1.580 & 0.212 & 0.072 & 1.063 \\
\hline S-PB-A7 & 21.017 & -1.011 & 0.912 & 0.989 & 0.382 & -0.116 & 0.209 & 0.111 & 0.980 \\
\hline S-PB-A11 & 33.902 & 0.195 & 1.770 & 1.017 & 0.242 & -0.749 & 0.169 & 0.258 & 1.014 \\
\hline S-PB-A13 & 36.005 & -0.140 & 0.188 & 1.031 & 0.161 & -0.389 & 0.255 & 0.060 & 1.075 \\
\hline S-PB-A14 & 22.728 & 0.112 & 0.487 & 0.976 & 0.228 & -0.709 & 0.201 & 0.035 & 1.040 \\
\hline S-PB-A15 & 49.339 & -0.102 & 0.098 & 0.952 & 0.221 & -0.373 & 0.273 & 0.036 & 1.016 \\
\hline S-PB-A19S & 51.839 & -1.494 & 0.422 & 0.981 & 0.300 & 0.050 & 0.238 & 0.140 & 1.124 \\
\hline S-PB-A19 & 38.488 & 0.079 & 0.476 & 0.966 & 0.138 & -0.821 & 0.275 & 0.183 & 0.965 \\
\hline S-PB-A22 & 29.505 & 0.672 & 0.827 & 0.901 & 0.285 & -0.164 & 0.699 & 0.155 & 1.076 \\
\hline S-PB-A23 & 31.656 & 0.145 & 1.008 & 0.987 & 0.183 & -0.668 & 0.185 & 0.088 & 0.972 \\
\hline S-PB-A25 & 39.730 & -0.899 & 1.061 & 1.013 & 0.257 & -0.113 & 0.198 & 0.188 & 1.022 \\
\hline S-PB-A28 & 34.262 & -0.806 & 0.359 & 0.996 & 0.395 & 0.092 & 0.217 & 0.040 & 1.022 \\
\hline S-PB-A30 & 42.843 & -0.432 & 0.808 & 1.001 & 0.234 & -0.254 & 0.173 & 0.102 & 1.124 \\
\hline S-PB-A31 & 18.337 & 1.561 & 0.110 & 1.052 & 0.540 & 0.550 & 0.296 & 0.045 & 1.182 \\
\hline S-PB-A32 & 41.111 & -1.215 & 0.331 & 0.947 & 0.233 & -0.073 & 0.250 & 0.063 & 1.110 \\
\hline S-PB-A33 & 40.320 & -0.211 & 0.060 & 1.000 & 0.179 & -0.396 & 0.195 & 0.105 & 1.024 \\
\hline S-PB-A34 & 30.069 & 0.130 & 1.214 & 0.994 & 0.287 & -0.361 & 0.141 & 0.073 & 1.005 \\
\hline S-PB-A36 & 36.170 & -0.076 & 0.955 & 0.995 & 0.270 & -0.412 & 0.214 & 0.128 & 1.029 \\
\hline S-PB-A38 & 56.661 & -0.010 & 0.121 & 0.949 & 0.163 & -0.516 & 0.208 & 0.165 & 1.420 \\
\hline S-PB-A39 & 44.764 & -0.864 & 0.735 & 0.973 & 0.242 & -0.160 & 0.218 & 0.523 & 1.188 \\
\hline S-PB-A27 & 35.739 & 0.392 & 1.283 & 0.823 & 0.433 & 0.262 & 0.276 & 0.137 & 1.170 \\
\hline S-PB-A5 & 26.111 & 0.362 & 0.460 & 0.969 & 0.405 & 0.190 & 0.213 & 0.055 & 1.075 \\
\hline S-PB-A4 & 24.817 & -2.029 & 0.185 & 0.976 & 0.391 & 0.041 & 0.281 & 0.057 & 1.157 \\
\hline S-PB-A16 & 27.397 & -0.460 & 0.086 & 0.961 & 0.445 & 0.107 & 0.307 & 0.043 & 1. \\
\hline
\end{tabular}

Tabla 3.-Valores estimados de los parámetros de la función de crecimiento, sensibilidad media (SM), varianza residual media de la serie ajustada (VM) y valor del índice (IND) en 46 individuos de Pinus silvestris de una parcela del área de Refalguerí (Puertos de Beceite, Tarragona). 


\begin{tabular}{lrrrrc} 
& \multicolumn{1}{c}{ ALFA } & BETA & GAMMA & DELTA & k \\
\hline Beta & -0.178 & & & & \\
Gamma & 0.123 & -0.032 & & & \\
Delta & -0.106 & 0.015 & -0.221 & & \\
k & -0.592 & $0.558^{*}$ & -0.177 & 0.006 & \\
v & -0.329 & $0.691^{*}$ & -0.252 & 0.021 & $0.748^{*}$
\end{tabular}

Tabla 4.-Matriz de correlación entre los valores estimados de los seis parámetros de la función de crecimiento para los 46 árboles de la parcela de Refalgueri (Puertos de Tortosa). $*=\mathrm{p}>0.001$.

Si este sencillo modelo conceptual fuera válido, equivaldría a transformar la ecuación (2) en la:

$$
\mathrm{R}_{\mathrm{t}}=\mathrm{K}+\mathrm{gt}^{\delta} \operatorname{con} \delta>0.5
$$

la mayor parte de los valores estimados para el parámetro $\delta$ en una amplia gama de individuos pertenecientes a diferentes especies (tabla 2) se sitúan en el rango de $0.50-1$.

Introduciendo el término de crecimiento alométrico final, la función (1) pasa a convertirse en:

$$
R_{1}=\alpha \cdot\left[1 \pm e^{(\beta-k t)}\right]^{-1 / v}+g t^{\delta}
$$

que permite ajustar las curvas de crecimiento indeterminado.

\section{ANÁLISIS DE LA FUNCIÓN}

La función (4) así resultante se ha utilizado para ajustar las series de crecimiento de los 46 árboles (Pinus silvestris) presentes en una parcela de $25 \times 25 \mathrm{~m}$. en el área de Refalgueri en los Puertos de Beceite (Tarragona). Los valores obtenidos en las estimaciones de los parámetros se presentan en la tabla 3. A partir de estos datos estimados, se ha calculado la matriz de correlaciones entre los parámetros (tabla 4).

Esta matriz muestra unos valores de correlación moderados. Las correlaciones más altas, entre $\mathrm{k} \mathrm{y} \vee(\mathrm{r}=0.748, \mathrm{p}>0.001)$, entre $\beta$ y $\mathrm{v}(\mathrm{r}=0.691$, $\mathrm{p}>0.001)$ y entre $\beta$ y k $(\mathrm{r}=0.558, \mathrm{p}>0.001)$ son, no obstante, muy inferiores a las obtenidas por CAUSTON y VENUS (1981) al describir el crecimiento de hojas individuales de trigo.

Un análisis de componentes principales sobre los seis parámetros de la función pone de manifiesto una clara estructura entre los mismos. La primera componente (figura 3 ) agrupa los parámetros $\beta, \mathrm{k}$ y v en la parte positiva del eje y el parámetro $\alpha$ cargando en la parte negativa del mismo (compárese con la correlación entre $\alpha \cup \mathrm{k} \mathrm{r}=-0.592$ ). Esta componente explica el 68.7 por cien- 


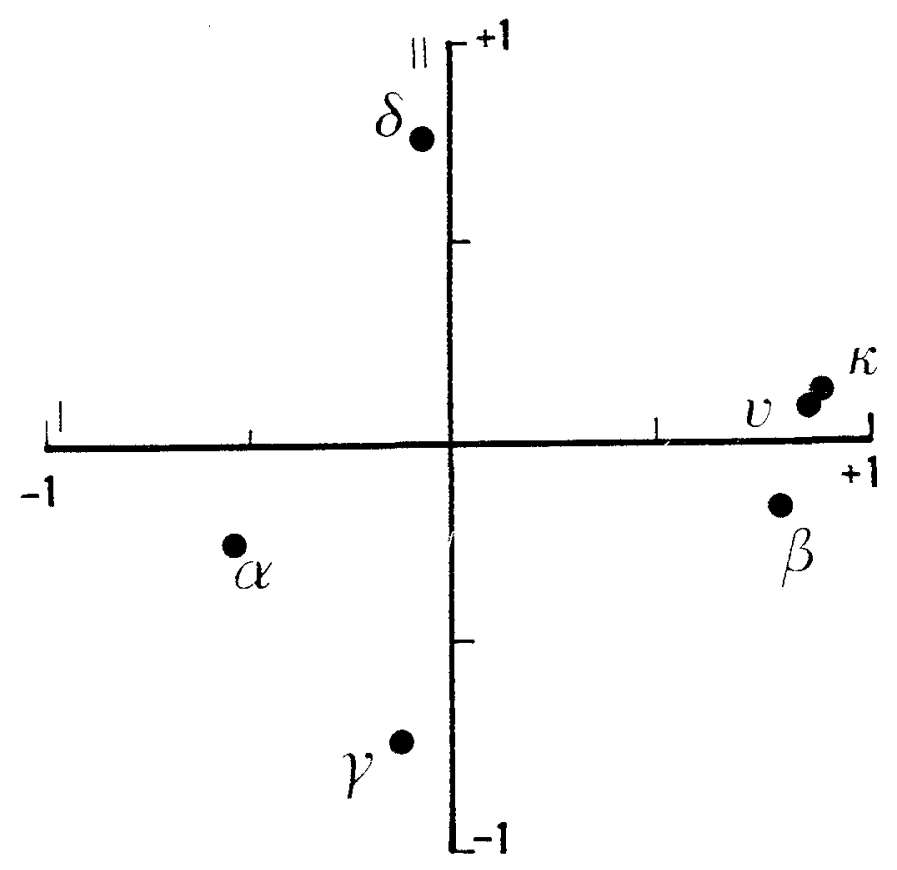

Figura 3.-Protección en el espacio de las dos primeras componentes principales de los seis parámetros de ajuste de la función de crecimiento para la parcela de Pinus sylvestris de Refalgueri (Puertos de Beceite). La primera componente, que explica un 68.7 por ciento de la varianza, pone de manifiesto la interdependencia entre los valores de beta, $\mathrm{k} y$ nu principalmente. Sobre la segunda componente, que explica el 31.3 por ciento restante de la varianza, se asocian los parámetros gamma y delta que definen la forma del crecimiento asintótico. Puesto que ambos bloques de variables cargan sobre dos componentes ortogonales de modo manifiesto, se puede considerar que la función describe el crecimiento como suma de dos componentes aproximadamente independientes.

to de la varianza en tanto que una segunda componente, que recoge los efectos de los parámetros g y $\delta$, que describen el crecimiento asintótico del final de la serie, explica un 31.3 por ciento de la varianza. Aceptando el resultado del análisis y llevando la interpretación más allá, podemos tratar de entender la función como la suma de dos componentes que se representan por los dos términos del segundo miembro de la función. El primer término explicaría el crecimiento de la fase inicial, hasta que el árbol llega a alcanzar una estructura funcional en equilibrio con el medio. De esto depende su crecimiento inicial, el momento en el que se alcanza el punto de inflexión y el tiempo necesario para alcanzar la asíntota. Una vez alcanzada ésta, el segundo término de la ecuación describe básicamente el crecimiento a partir de dicho punto que se puede aproximar bien por una función potencial del tipo de la función (3) con $\delta$ muy próxima a la unidad (ver tablas 1,2 y 3 ). 
La figura 1 ayudará a comprender el sentido de la función (4) y de la base conceptual del algoritmo utilizado para estimar los valores de los parámetros que presentaremos más adelante. Supongamos que los valores acumulados de la serie que queremos ajustar vienen representados por la curva (A). El primer término de la ecuación (4) - que constituye la función de Richard propiamente dicha para un crecimiento de tipo determinado- describe en realidad un crecimiento del tipo representado por (B). El valor de $\alpha$, por lo tanto, corresponde al radio del árbol en el momento Ta en el que el árbol alcanza el crecimiento asintótico a partir del cual, y puesto que el valor del primer término de la función se hace constante, el crecimiento viene definido por el segundo término que representa el crecimiento alométrico que hemos discutido previamente.

El punto de inflexión se alcanza en el instante $T_{i}$ que viene dado por:

$$
\mathrm{T}_{\mathrm{i}}=\frac{\beta-\ln ( \pm \mathrm{v})}{\mathrm{k}}
$$

Lá tasa de crecimiento $r$ se estima como:

$$
r=\frac{k}{v+1}
$$

y la tasa de crecimiento absoluto $\mathrm{R}$ viene dada por:

$$
\mathrm{R}=\frac{\alpha \cdot \mathrm{k}}{2(\mathrm{v}+2)}
$$

que es una estima promediada del incremento anual de los anillos de crecimiento.

\section{ESTABILIDAD DE LAS ESTIMACIONES DE LOS PARÁMETROS}

La utilización de la función requiere conocer su comportamiento en las estimaciones de los parámetros y, muy en particular, la estabilidad de tales estimaciones. En términos sencillos diremos que las estimaciones son estables si obtenemos valores estimados aproximadamente constantes, independientemente de la longitud de la serie utilizada siempre y cuando ésta haya alcanzado el crecimiento asintótico. En otras palabras, si al crecer el árbol y añadir nuevos anillos a la serie, una vez alcanzada la asíntota, se lograran valores estimados diferentes para los parámetros, estaríamos ante un algoritmo de estimación de dudosa utilidad.

El procedimiento seguido para evaluar la estabilidad ha consistido en estimar sucesivamente los valores de los parámetros que corresponden a una serie (la del core S-PB-A7) de 52 años. Las estimaciones se han repetido sucesivamente suprimiendo cada vez un año del final de la serie hasta quedar reducida a sólo 20 años. Los resultados obtenidos en las 33 estimaciones sucesivas 


\begin{tabular}{|c|c|c|c|c|c|c|c|c|c|c|c|}
\hline & & $\beta$ & $\varphi$ & $\delta$ & $\kappa$ & $v$ & $5 \mathrm{M}$ & $M$ & IND & Ti & \\
\hline & & & & 90 & 383 & 3 & 0.21 & 0.11 & & & \\
\hline & & & & & & & & & & & \\
\hline & & & & & & & & & & & \\
\hline & 4 & & & & & & & & & & \\
\hline & & & & & & & & & & & \\
\hline & & & & & & & & & & & \\
\hline & & & & & & & & & & & \\
\hline & & & & & & & & & & & \\
\hline & & & & & & & & & & & \\
\hline & & & & & & & & & & & \\
\hline & & 3 & & & & & & & & & \\
\hline & & 1.056 & $\boldsymbol{U}$ & & & & & & & & \\
\hline & & & & & & & & & & & \\
\hline & & & & & & & & & & & \\
\hline & & & & & & & & & & & \\
\hline & & 1.0 & & & & & & & & & \\
\hline & & & & & & & & & & & \\
\hline & & & & & & & & & & & \\
\hline & & & & & & & & & & & \\
\hline & & 6 & 53 & & & & & & & & \\
\hline & & & & & & & & & & & \\
\hline & & & & & & & & & & & \\
\hline & & & & & & & & & & & \\
\hline & & & & & & & & & & & \\
\hline & & -0. & & & & & & & & & \\
\hline & & -06 & & & & & & & & & \\
\hline & & 1 & & & & & & 17 & & & \\
\hline & & & & & & & & & & & \\
\hline & & & & & & & & & & & \\
\hline & & & & & & & & & & & \\
\hline & & -0 & 055 & 923 & & & 0.2 & 0.21 & & & \\
\hline & & & 062 & & & & & 0. & & & \\
\hline & & & 00 & & & & & & & & \\
\hline
\end{tabular}

Tabla 5.-Valores estimados de los parámetros de la función de crecimiento, sensibilidad media (SM), varianza residual media (VM), índices medios (IND), tiempo de inflexión (Ti) y tiempo en el que se alcanza la asíntota (Ta) de un árbol de 52 años de la parcela de Refalguerí al que se le suprimen sucesivamente los anillos de crecimiento finales hasta dejar la serie reducida a 20 años. 
así realizadas se presentan en la tabla 5. Como, los valores estimados para el parámetro $\alpha$, que representa el valor del radio del árbol en el momento de alcanzar la asíntota, varía ligeramente desde $21.00 \mathrm{~mm}$ con la serie completa de 52 años a 22.6 cuando la serie se reduce a 20 años. Los valores del núcleo formado por los parámetros $\beta, \mathrm{k}$ y v, que como vimos están fuertemente correlacionados, se comprenden mejor si consideramos el valor de los parámetros secundarios $T_{i}, T_{a}, R_{i}$ y $D_{a}$ (siendo $D_{a}=2 R_{a}$, es decir el diámetro del árbol sin corteza en el momento de alcanzar el crecimiento asintótico). En todos los casos que resultan de la combinación de los parámetros, la estabilidad de los valores estimados es muy notable, a pesar de que, al reducir la longitud de la serie, los primeros valores, siempre más fluctuantes aumentan la varianza media de la serie que pasa de 0.11 con 56 valores a 0.23 con 20 valores.

\section{ESTIMACIÓN DE LOS PARÁMETROS DE LA FUNCIÓN DE CRECIMIENTO}

El programa GROWTH desarrollado por uno de los autores en dos versiones diferentes (PL/1 y BASIC), permite estimar los valores de los parámetros de la función a partir de los espesores de los anillos de crecimiento anual. El procedimiento utilizado en el algoritmo de estimación se resume brevemente a continuación.

Se comienza estimando el valor de la curva alométrica asintótica por mínimos cuadrados aplicados sobre las transformaciones logarítmicas de ambos ejes, el del tiempo y el de los valores acumulados de crecimiento. Una vez conocidos los valores de $\mathrm{g}$ y de $\delta$, la función original se transforma haciendo:

$$
\mathrm{Q}(\mathrm{t})=\mathrm{R}(\mathrm{t})-\mathrm{gt} \mathrm{t}^{\delta}
$$

de modo que la nueva función $\mathrm{Q}(\mathrm{t})$ tenga una asíntota horizontal. GROWTH comprueba si la pendiente de esta función es $0 \mathrm{y}$, si es necesario, corrige el valor de $\mathrm{g}$. Además, ningún valor de $\mathrm{Q}(\mathrm{t})$ puede ser igual o inferior a 0 . De ser así, el valor de g se considera sobreestimado y se somete a una nueva corrección.

Conocido el valor de $\mathrm{g}$ se busca el valor de $\alpha$ que no es otro que la ordenada en el origen de la función alométrica haciendo:

$$
\alpha=\mathrm{R}(\mathrm{n})-\mathrm{gt}^{\delta}
$$

donde $\mathrm{n}$ es el número de años que cubre la serie experimental cuyos parámetros se buscan.

El valor de $\mathrm{v}$ se obtiene mediante un proceso iterativo de aproximaciones sucesivas, buscando el valor que soluciona la ecuación:

$$
\mathrm{R}(\mathrm{i})=\alpha(\mathrm{v}+1)^{(-1 / \mathrm{v})}+\mathrm{gt}^{\delta}
$$


siendo R(i) el valor del radio del árbol en el momento de la inflexión. Conocido v se calcula $\mathrm{k}$ resolviendo:

$\frac{\operatorname{INC}(\mathrm{R}(\mathrm{i}))}{\operatorname{INC}(\mathrm{T}(\mathrm{i}))}=\frac{\mathrm{k} \cdot \mathrm{R}(\mathrm{i})}{\mathrm{v} \cdot \alpha \mathrm{v}}(\alpha \mathrm{v}-\mathrm{R}(\mathrm{i}) \mathrm{v})+\mathrm{g}$

donde INC (R(i)) es el incremento del radio en el momento de la inflexión.

El valor de $\beta$ se obtiene directamente resolviendo:

$$
\mathrm{T}(\mathrm{i})=\frac{\beta-\log \mathrm{v}}{\mathrm{k}}
$$

Información más detallada sobre GROWTH se puede obtener directamente de los autores.

\section{AGRADECIMIENTOS}

Algunas muestras de las que se han obtenido una parte de las series presentadas en la tabla 2 , fueron recogidas en el campo y analizadas por el Dr. R. Génova a quien quedamos profundamente agradecidos. 


\section{BIBLIOGRAFÍA}

ANIOL, R. 1983: Procedures and statistics in dendrochronology. En: Dendrochronology and Archaeology in Europe. (Eckstein, D et al. Eds.) Wiedebusch, Hamburg. pp.: 21-30.

BAILLIE, M. G. R. 1977: An oak chronology for South Central Scotland. Tree-ring Bulletin. 37: 33-44.

BERGER, A.; GUIOT, J.; MATHIEU, L. y MUNAUT, A. V. 1979. Tree-rings and climate in Morocco. Tree-ring Bulletin. 39: 61-76.

CAUSTON, D. R.; ELIAS, C. O. y HADLEY, P. 1978: Biometrical Studies of plant growth. I. The Richards function and its application in analising the effects of temperature on leaf growth. Plant, Cell and Environment, 1: 163-168.

CAUSTON, D. R. y VENUS, J. C. 1981: The Biometry of plant growth. Edward Arnold (Publishers). Londres. $307 \mathrm{pp}$.

FRITTS, H. C. 1976: Tree-rings and climate. Academic Press. Londres.

GÉNOVA, R. 1987: Análisis y significado de los anillos de crecimiento de dos especies forestales: Pinus uncinata y Pinus syilvestris, en la Península Ibérica. Tesis Doctoral, Universidad de Barcelona.

GRACIA, C. A. e IBÁÑEZ, J. J. 1986: Efectos de la competencia sobre la producción de masas forestales. En: Bases ecologiques per a la gestio ambiental. Diputació de Barcelona. pp.: 56-57.

GRAYBILL, D. A. 1982: Chronology development and analysis. En: climate from tree-rings. (Hughes M. K. et al. edits.) Cambridge Univ. Press. pp: $2^{\mathrm{a}}-28$.

GUTIÉRREZ, E. 1987: Dendrocronología de Fagus silvatica, Pinus uncinata y Pinus syilvestris en Cataluña. Tesis doctoral, Universidad de Barcelona.

RICHARDS, F. J. 1959: A flexible growth function for empirical use. Journal of experimental Botany, 10: 290-300.

STOKES, M. A. y SMILEY, T. L. 1968: An introduction to tree-ring dating. University of Chicago Press.

ZUCCHINI W. y HIEMSTRA, L. A. V. 1983: A note on the relationship between annual rainfall and tree-ring indices for one site in South Africa. Water 4: 153-154. 